\title{
AN EXTREMALLY DISCONNECTED DOWKER SPACE
}

\author{
ALAN DOW AND JAN VAN MILL
}

ABSTRACT. We give an example of an extremally disconnected Dowker space. Our basic tool is that every $P$-space can be $C^{*}$-embedded in an extremally disconnected compactum.

0. Introduction. A Dowker space is a normal space $X$ for which $X \times I$ is not normal, where $I$ denotes the closed unit interval $[0,1]$. Dowker spaces are hard to get. Under various set theoretic hypotheses, Dowker spaces with many additional properties have been constructed. In ZFC only one construction of a Dowker space is known, see Rudin [R].

Hardy and Juhász [HJ] asked whether extremally disconnected Dowker spaces exist, ${ }^{1}$ where a space $X$ is called extremally disconnected if the closure of each open subspace of $X$ is given again open. They also announced that Wage had constructed such a space; however that turned out to be incorrect. The aim of this note is to construct an extremally disconnected Dowker space in ZFC. The reader who hopes that we found a new way of constructing Dowker spaces in ZFC will be quite disappointed. What we do is simply modify Mary Ellen Rudin's [R] Dowker space so that it becomes extremally disconnected. Our technique is to show that every $P$-space can be $C^{*}$-embedded in some compact extremally disconnected space, thus generalizing results in [BSV and $\mathbf{v D}$ ].

1. Preliminaries. Let $X$ be a compact space and let $R O(X)$ be the Boolean algebra of regular open subsets of $X$. The Stone space of $R O(X)$ is denoted by $E X$ and is called the projective cover of $X$. The function $\pi: E X \rightarrow X$ defined by

$$
\{\pi(u)\}=\bigcap_{U \in u} \bar{U}
$$

is easily seen to be continuous, onto and irreducible, i.e. if $A \subseteq E X$ is a proper closed subpsace, then $\pi(A) \neq X$. Since $R O(X)$ is complete, $E X$ is extremally disconnected. If $h: X \rightarrow X$ is a homeomorphism, then the function $e h: E X \rightarrow E X$ defined by $e h(u)=\{h(U): U \in u\}$ is easily seen to be a homeomorphism such that $\pi \circ e h=$ $h \circ \pi$. The reader is encouraged to check this, since we use this later. For a recent survey on projective covers, see Woods [W]. By a result of Efimov [E], every extremally disconnected compactum embeds in the Čech-Stone compactification $\beta \kappa$

Received by the editors December 10, 1981 and, in revised form, April 13, 1982.

1980 Mathematics Subject Classification. Primary 54D35.

$K e y$ words and phrases. Dowker space, $P$-space, $C^{*}$-embedding, $\beta X$.

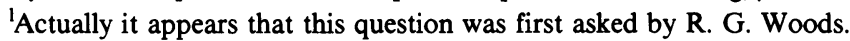


of some cardinal $\kappa$, where $\kappa$ is given the discrete topology. As usual, we call a space $X$ a $P$-space if every $G_{\delta}$ in $X$ is open. If $X$ is a Tychonoff space, then $\beta X$ denotes the Cech-Stone compactification of $X$. A subspace $Y \subseteq X$ is said to be $C^{*}$-embedded in $X$ provided that every map $f: Y \rightarrow I$ extends to a map $\bar{f}: X \rightarrow I$. Our terminology is standard. $w(X)$ denotes the weight of a space $X$.

2. Embedding $P$-spaces in $\beta \kappa$. In this section we show that if $X$ is a $P$-space, then $\beta X$ can be embedded in the Čech-Stone compactification of some discrete space. Obviously, this is equivalent to the statement that every $P$-space can be $C^{*}$ embedded in some extremally disconnected compact space.

To this end, let $X$ be a $P$-space. Since $X$ is strongly zero-dimensional, we may assume that $\beta X \subseteq 2^{\kappa}$ for certain $\kappa$. Take $p \in 2^{\kappa}$. The map $g_{p}: 2^{\kappa} \rightarrow 2^{\kappa}$ defined by $g_{p}(x)=x+p$ lifts to a map $e g_{p}: E\left(2^{\kappa}\right) \rightarrow E\left(2^{\kappa}\right)$, see $\$ 1$. The homeomorphism $e g_{p}$ will be called $h_{p}$ for short.

2.1. LEMMA. If $U \in R O\left(2^{*}\right)$ then there exist a countable collection $\left\{F_{n}: n \in \omega\right\}$ of finite subsets of $\kappa$ and elements $\delta_{n}$ of $2^{F_{n}}$, for $n \in \omega$, such that $\cup_{n \in \omega}\left(\cap_{i \in F_{n}} \pi_{i}^{\leftarrow}(\delta(i))\right)$ is a dense subset of $U$ (where $\pi_{i}$ is the ith projection map).

Proof. As is well known, $2^{\kappa}$ is ccc (families of pairwise disjoint open sets are countable) and the collection $\mathscr{B}=\left\{\bigcap_{i \in F^{-}} \pi_{i}^{-}(\delta(i)): F \subseteq \kappa\right.$ is finite and $\left.\delta \in 2^{F}\right\}$ is a base for the topology of $2^{\kappa}$. Choose a maximal cellular collection, $\mathcal{C} \subseteq \mathscr{B}$, of subsets of $U$. Clearly $\mathcal{C}$ is countable and $\cup \mathcal{C}$ is dense in $U$. Take $\left\{F_{n}: n \in \omega\right\}$, finite subsets of $\kappa$, and $\delta_{n} \in 2^{F_{n}}$, for $n \in \omega$, so that $C=\left\{\bigcap_{i \in F_{n}} \pi_{i}^{-}\left(\delta_{n}(i)\right): n \in \omega\right\}$.

If $U \in R O\left(2^{\kappa}\right)$ and $\left\{F_{n}: n \in \omega\right\}$ is chosen as in 2.1 , then we say that $U$ is determined by $D=\cup F_{n}$. The following lemma follows trivially from the definition of $g_{p}$ for $p \in 2^{\kappa}$.

2.2. LEMMA. If $i \in \kappa$ and $\pi_{i}(p)=\pi_{i}(q)$ for $p, q \in 2^{\kappa}$ then $g_{p}\left(\pi^{-}(\delta)\right)=g_{q}\left(\pi^{\leftarrow}(\delta)\right)$ for $\delta \in\{0,1\}$.

2.3. Lemma. If $U \in R O\left(2^{\kappa}\right), U$ is determined by $D$ and $p, q \in 2^{\kappa}$ are such that $p \uparrow D=q \uparrow D$, then $g_{p}(U)=g_{q}(U)$.

Proof. Let $\left\{F_{n}: n \in \pm \omega\right\}$ and $\left\{\delta_{n}: n \in \omega\right\}$ with $D=\cup_{n \in \omega} F_{n}$ be as in 2.1. From 2.2 , it follows that $g_{p}\left(\bigcap_{i \in F_{n}} \pi_{i}^{-}\left(\delta_{n}(i)\right)\right)=g_{q}\left(\bigcap_{i \in F_{n}} \pi_{i}^{\leftarrow}\left(\delta_{n}(i)\right)\right)$ for each $n \in \omega$, and therefore

$$
g_{p}\left(\bigcup_{n \in \omega}\left(\bigcap_{i \in F_{n}} \pi_{i}^{-}\left(\delta_{n}(i)\right)\right)\right)=g_{q}\left(\bigcup_{n \in \omega}\left(\bigcap_{i \in F_{n}} \pi_{i}^{-}\left(\delta_{n}(i)\right)\right)\right) .
$$

Since the image under $g_{p}$ and $g_{q}$ of a dense subset of $U$ is the same, $g_{p}(U)=g_{q}(U)$.

Take a point $u_{0} \in \pi^{\leftarrow}(\mathbf{0})$, where $\mathrm{O}$ denotes the identity of $2^{\kappa}$. If $p \in X$, let $u_{p}=h_{p}\left(u_{0}\right)$. Observe that

$$
\pi\left(u_{p}\right)=\pi\left(h_{p}\left(u_{0}\right)\right)=g_{p}\left(\pi\left(u_{0}\right)\right)=g_{p}(\mathbf{0})=p,
$$


whence $u_{p} \in \pi^{\leftarrow}(p)$. If $U \in R O\left(2^{\kappa}\right)$ then $\overline{h_{p}\left(\pi^{\leftarrow}(u)\right)}=\overline{\pi^{\leftarrow}\left(g_{p}(U)\right)}$ and from this it follows that $u_{p}=\left\{g_{p}(U): U \in u_{0}\right\}$. Note also that since $g_{p} \circ g_{p}=\mathrm{id}, u_{p}=\{U$ : $\left.g_{p}(U) \in u_{0}\right\}$. Let $P=\left\{u_{p}: p \in X\right\}$.

\subsection{Lemma. The function $\pi \uparrow P: P \rightarrow X$ is a homeomorphism.}

Proof. For convenience, put $f=\pi \uparrow P$. Then $f$ is clearly one-to-one, onto and continuous. It therefore suffices to show that $f$ is open. Basic open sets of $P$ are of the form $\tilde{U}$, where $U \in R O\left(2^{\kappa}\right)$ and $\tilde{U}=\left\{u_{p} \in P: U \in u_{p}\right\}$. Choose $p \in f(\tilde{U})$ and let $U$ be determined by $D$. Let $Z=\{q \in X: p \uparrow D=q \uparrow D\}$. By 2.3, $g_{p}(U)=g_{q}(U)$ and, therefore, $u_{q} \in \tilde{U}$ by the above remarks, for each $q \in Z$. Now $Z=X \cap$ $\bigcap_{i \in D} \pi_{i}^{\leftarrow}\left(\pi_{i}(p)\right)$ is a $G_{\delta}$-set of $X$ and therefore open in $X$. Since $p \in Z$ and $Z \subseteq f(\tilde{U})$, we conclude that $f(\tilde{U})$ is a neighborhood of $p$.

The closure of $P$ in $E\left(2^{\kappa}\right)$ is a compactification of $P$ which is clearly homeomorphic to $\beta X$ since $\beta X$ is the largest compactification of $X$. This completes the proof, since by Efimov's result $(\S 1), E\left(2^{\kappa}\right)$ can be embedded in the Čech-Stone compactification of a discrete space.

The reader can easily verify that in fact we have shown that if $X$ is a $P$-space of weight $\kappa$ then $\beta X$ can be embedded in $\beta\left(2^{\kappa}\right)$ (here $2^{\kappa}$ has the discrete topology of course).

3. The example. The Dowker space $R$ constructed in Rudin $[\mathbf{R}]$ is a $P$-space. By the results in $\S 2, \beta R$ embeds in $\beta \kappa$ for certain $\kappa$. Since $\beta \kappa$ embeds in $\beta \kappa-\kappa$, we may assume that $\beta R \subseteq \beta \kappa-\kappa$. Put $X=\kappa \cup R$. Since each dense subspace of an extremally disconnected space is extremally disconnected, $X$ is extremally disconnected. Also, $R$ is closed in $X$ which implies that $X \times I$ is not normal since $R \times I$ is not normal. Since $\kappa$ is discrete, a moment's reflection shows that $X$ is normal iff disjoint closed subsets of $R$ have disjoint neighborhoods in $X$. Let $A, B \subseteq R$ be closed and disjoint. Since the closure of $R$ in $\beta \kappa$ is $\beta R, A$ and $B$ have disjoint closures in $\beta R$, hence they have disjoint neighborhoods in $\beta \kappa$. We conclude that $X$ is normal and consequently that $X$ is an extremally disconnected Dowker space.

Observe that our example, in particular, is an example of a normal extremally disconnected space which is not paracompact. Such a space was earlier constructed by Kunen [K].

4. Remarks. (1) The technique used in $\$ 2$ is a modification of a technique due to Balcar, Simon and Vojtás [BSV] and, independently, Kunen, and Shelah. They observe that if $p_{\alpha}$ is the point of $2^{\kappa}$ with value 1 only in the point $\{\alpha\}$ then the set $\left\{u_{p_{\alpha}}: \alpha<\kappa\right\} \subseteq E\left(2^{\kappa}\right)$ is discrete and each neighborhood of $u_{0}$ contains all but countably many points of $\left\{u_{p_{\alpha}}: \alpha<\kappa\right\}$ (the notation is as in \$2).

(2) van Douwen [vD] used the technique described in (1) to prove the important result that every $P$-space embeds in $\beta \kappa$ for certain $\kappa$. His proof goes as follows. Let $X=\left\{u_{p}: p \in 2^{\kappa}\right\}$. Then $X$ considered to be a subspace of $E\left(2^{\kappa}\right)$ with the $G_{\delta}$ topology, is homeomorphic to $2^{\kappa}$ with the $G_{\delta}$ topology. Moreover, $E\left(2^{\kappa}\right)$ with the $G_{\delta}$ topology embeds in $E\left(2^{\kappa}\right)$. Consequently, $2^{\kappa}$ with the $G_{\delta}$ topology embeds in $E\left(2^{\kappa}\right)$ and hence in $\beta\left(2^{\kappa}\right)$. If $P \subseteq 2^{\kappa}$ is a $P$-space, then $P$ is homeomorphic to $P$ considered 
to be a subspace of $2^{\kappa}$ with the $G_{\delta}$ topology. Consequently, $P$ embeds in $\beta\left(2^{\kappa}\right)$. Our results in $\$ 2$ were motivated by these ideas but our construction is much simpler and proves more since our embeddings of $P$-spaces are embeddings of $C^{*}$-embedded subspaces of $E\left(2^{\kappa}\right)$ and this made our construction work.

\section{REFERENCES}

[BSV] B. Balcar, P. Simon and P. Vojtás, Refinement properties and extensions of filters in Boolean algebras, Trans. Amer. Math. Soc. 267 (1981), 265-283.

[vD] E. K. van Douwen (in preparation).

[E] B. A. Efimov, Extremally disconnected compact spaces and absolutes, Trans. Moscow Math. Soc. 23 (1970), 243-285.

[HJ] K. Hardy and I. Juhász, Normality and the weak cb property, Pacific J. Math. 64 (1976), 167-172.

[K] K. Kunen, An extremally disconnected space, Notices Amer. Math. Soc. 24 (1977), A-263.

[R] M. E. Rudin, $A$ normal space $X$ for which $X \times I$ is not normal, Fund. Math. 73 (1971), 179-186.

[W] R. G. Woods, A survey of absolutes of topological spaces, Top. Structures II, Math. Centre Tracts 116 (1979), 323-362.

SUbFaculteit Wiskunde, VRije Universiteit, De BoelelaAn 1081, Amsterdam, The NetherLandS 